\title{
Efficacy of two low-dose oral tylosin regimens in controlling the relapse of diarrhea in dogs with tylosin-responsive diarrhea: a prospective, single-blinded, two-arm parallel, clinical field trial
}

Susanne Kilpinen*, Thomas Spillmann and Elias Westermarck

\begin{abstract}
Background: Despite its wide acceptance as a treatment for canine chronic enteropathies, the macrolide antibiotic tylosin lacks official oral dosage recommendations. Not even textbooks share consensus about the dose; daily recommendations vary from 25 to $80 \mathrm{mg} / \mathrm{kg}$ and dosing intervals from one to three times daily.

The objective of this prospective, single-blinded, two-arm parallel, clinical field trial was to determine whether doses of $5 \mathrm{mg} / \mathrm{kg}$ or $15 \mathrm{mg} / \mathrm{kg}$ tylosin administered orally once daily for seven days would have a similar effect on fecal consistency in diarrhea relapses to that of a $25 \mathrm{mg} / \mathrm{kg}$ dose of tylosin administered once daily for seven days, a dosage that has proved effective in controlling canine tylosin-responsive diarrhea (TRD). A further objective was to compare the efficacy of the $5 \mathrm{mg} / \mathrm{kg}$ and $15 \mathrm{mg} / \mathrm{kg}$ tylosin dosages. Fifteen client-owned dogs diagnosed with TRD that had responded to a dose of $25 \mathrm{mg} / \mathrm{kg}$ tylosin once daily for seven days were enrolled in the study. After a relapse of diarrhea the dogs were allocated into two groups receiving tylosin orally in doses of either $5 \mathrm{mg} / \mathrm{kg}$ or $15 \mathrm{mg} / \mathrm{kg}$ once daily for seven days. The owners were blinded to the dosage. The elimination of diarrhea was the main criterion in assessing treatment success. The mean fecal consistency score of the last three treatment days for all dosages, including $25 \mathrm{mg} / \mathrm{kg}$, as evaluated by the owners according to a standardized fecal scoring system, served as the primary outcome measures.

Results: All eight dogs responded to the $5 \mathrm{mg} / \mathrm{kg}$ dose, and six of seven dogs responded to the $15 \mathrm{mg} / \mathrm{kg}$ dose. The mean fecal consistency scores at the $25 \mathrm{mg} / \mathrm{kg}$ tylosin dosage were no significantly different from scores at the $5 \mathrm{mg} / \mathrm{kg}$ or $15 \mathrm{mg} / \mathrm{kg}$ tylosin dosages $(P=0.672, P=0.345)$.

Conclusions: Interestingly, 14/15 (93\%) of the dogs responding to a dose of $25 \mathrm{mg} / \mathrm{kg}$ tylosin once daily for seven days also responded to the lower dosages at diarrhea relapse. The data indicate that a suitable dose of tylosin for treating diarrhea relapse in canine TRD could be as low as $5 \mathrm{mg} / \mathrm{kg}$ once daily for seven days.
\end{abstract}

Keywords: Dosage, Fecal scoring system, Recurrent diarrhea, Antibiotic responsive diarrhea, Chronic enteropathy

* Correspondence: susanne.kilpinen@helsinki.fi

Department of Equine and Small Animal Medicine, Faculty of Veterinary

Medicine, University of Helsinki, P.O. Box 57, Helsinki Fl-00014, Finland 


\section{Background}

Tylosin, a member of the macrolide group of antibiotics, is widely used in the treatment of canine chronic diarrhea of unknown etiologies [1,2]. Currently, oral tylosin treatment is not approved for the treatment of canine enteritis, but it may be prescribed as an ex tempore drug, dispensed by the pharmacy on request. Consequently, no official recommendations are available for the oral dosage regimen in dogs, and the literature shows that individual authors' recommended dosages differ.

In 1976, Van Kruiningen drew attention to the usefulness of tylosin in treating enterocolitis in dogs in a study about the clinical efficacy of tylosin in canine inflammatory bowel disease [3]. This study comprised 27 dogs receiving a daily oral dosage of tylosin between 11-200 mg/ $\mathrm{kg}$. The dosing interval was twice daily, and the duration of treatment varied from one week to five years. Two decades later, a casecontrol study involved seven pet dogs with secondary chronic diarrhea due to naturally occurring exocrine pancreatic insufficiency. The dogs received tylosin for 5-7 days at a dose of $15 \mathrm{mg} / \mathrm{kg}$ twice daily as an adjunctive medication to the pancreatic enzyme [4]. In 2005, Westermarck et al. introduced the term tylosin-responsive diarrhea (TRD) in dogs in a case series involving nine client-owned dogs suffering from recurrent diarrhea and responding to tylosin at a dose of 6-16 mg/kg once daily for two weeks [5]. Characteristic of TRD was that the dogs with recurrent diarrhea, for which no underlying cause could be found, usually responded to tylosin within a few days. The stool remained normal as long as treatment continued, but diarrhea recurred in many dogs within weeks of discontinuing the treatment. After initiating a repeated course, however, the dogs' response to tylosin was as good as the initial treatment, even after several treatments.

A recent randomized controlled trial of the effect of tylosin on fecal consistency in dogs with recurrent diarrhea revealed that tylosin proved significantly more effective than placebo. At a dose of $25 \mathrm{mg} / \mathrm{kg}$ once daily for seven days, tylosin proved effective in controlling diarrhea in 20 client-owned dogs [6].

However, despite the wide use and acceptance of tylosin in the treatment of chronic enteropathies in dogs, a clear consensus about the dosage regimen is lacking. In textbooks, the dose recommendations for tylosin in dogs with chronic diarrhea vary considerably, with daily dosages ranging from 25 to $80 \mathrm{mg} / \mathrm{kg}$, and dosing intervals from one to three times daily $[2,7,8]$. These textbooks contain little information about the original source of this information. To date, no published dosage determination studies are available on the oral treatment of dogs with chronic diarrhea by tylosin.

Therefore, the primary objective of the current study was to determine whether tylosin doses of $5 \mathrm{mg} / \mathrm{kg}$ or $15 \mathrm{mg} / \mathrm{kg}$ administered orally once daily for seven days have a similar effect on the fecal consistency in diarrhea relapses to that of $25 \mathrm{mg} / \mathrm{kg}$ dose of tylosin once daily for seven days, a dosage that has proved effective in controlling canine TRD. A further objective was to compare the efficacy of the $5 \mathrm{mg} / \mathrm{kg}$ and $15 \mathrm{mg} / \mathrm{kg}$ dosages.

Secondary objectives were to assess the recurrence rate and the time to recurrence of diarrhea at a tylosin dosage of $25 \mathrm{mg} / \mathrm{kg}$ once daily over a seven-day treatment duration. Further objectives were to investigate the influence of the three different tylosin dosages on the time to the cessation of diarrhea.

\section{Methods}

\section{Trial design, compliance and ethics approval}

This trial was designed according the guidelines of evidence-based medicine [9] and reported utilizing the Consolidated Standards of Reporting Trials (CONSORT) [10-13]. The study design is a prospective, single-blinded, two-arm parallel clinical dosage determination field trial. The study complied with the European Economic Community Guideline on the demonstration of efficacy for veterinary medicinal products containing antimicrobial substances. Both the Ethics Committee for Animal Experiments of the University of Helsinki in Finland and the National Animal Experiment Board in Finland approved the study protocol. The owners provided their written informed consent in which they agreed to participate with their dog.

\section{Study population, inclusion and exclusion criteria}

This study was conducted at the Small Animal Teaching Hospital, Faculty of Veterinary Medicine, University of Helsinki, Finland. The study population was recruited from the patient material of a placebo-controlled, randomized, double-blinded, prospective clinical trial on the effectiveness of oral tylosin in treating recurrent diarrhea in dogs, that was conducted just prior to this current dosage determination trial [6]. Dogs were considered eligible for the oral tylosin dosage determination trial if they responded to a tylosin treatment at a dosage of $25 \mathrm{mg} / \mathrm{kg}$ once daily for seven days in the aforementioned trial. Inclusion criteria were that the dogs were at least six months old and had been suffering from recurrent diarrhea responding to tylosin treatment. They were not allowed to have received systemic corticosteroids, nonsteroidal anti-inflammatory drugs, or antibiotics other than tylosin in the 90 days preceding the tylosin dosage determination trial. Lactating bitches were excluded, as were dogs with evidence of systemic or organ-related disease that could secondarily cause diarrhea.

The dogs underwent an initial examination prior to enrollment in the tylosin effectiveness trial to ensure that they met the selection criteria. In brief, the initial 
examination covered the dogs' clinical history and physical examination, blood samples for a complete blood count and serum biochemical profile, urinalysis, fecal analysis for endoparasites and enteropathogenic bacteria, and gastroduodenoscopy with mucosal biopsies from the duodenum. The protocol and the results of the initial examination have been published previously [6].

\section{Outcome measures}

The primary objective of the current trial was to assess whether daily doses of $5 \mathrm{mg} / \mathrm{kg}$ or $15 \mathrm{mg} / \mathrm{kg}$ tylosin at a once daily dosing interval for seven days would have similar effects on the fecal consistency in diarrhea relapses to that of an initial dose of $25 \mathrm{mg} / \mathrm{kg}$ tylosin once daily for seven days in these dogs, a dosage that has proved effective in controlling canine TRD [6]. Further to assess whether the tylosin dose $5 \mathrm{mg} / \mathrm{kg}$ was as effective as the $15 \mathrm{mg} / \mathrm{kg}$ dose. To evaluate this, the elimination of diarrhea served as the main criterion. The elimination of diarrhea was fulfilled when noting that on three consecutive days the fecal score for each of the dog's feces was and remained three or less. A mean fecal consistency score assigned during the last three days of the seven-day treatment period served as the primary outcome measure. To determine the mean fecal consistency score, the owners evaluated and recorded throughout the study the consistency of each of their dog's stool according to a standardized fecal scoring system previously established by Moxham [14]. This kind of fecal scoring system has proved useful in evaluating treatment success in diarrhea patients [5,6,15-17]. In brief, the fecal scoring system is based on a nine-point scale, consisting of scores from one to five, with half-point intervals. To ensure uniformity in the fecal consistency scoring, it comprises both a verbal description and a visual picture for each of the fecal consistency scores. Scores 1 and 1.5 represented feces of a hard and dry consistency. Scores 2 and 2.5 indicated well-formed feces that left no mark when picked up. A score of 3 represented feces of a slightly moist consistency, whereas score 3.5 represented feces of a moist consistency, but which still had some definite form. Scores 4 through 5 described feces of poor consistency.

To evaluate whether the tylosin dosage was effective, the investigator calculated a mean fecal consistency score of the scores assigned for each of the dog's feces during the last three days of the seven-day treatment period based on the owner's records. A responder was defined as having a mean fecal consistency score of three or less; a nonresponder had a score of more than three. The owners were unaware of these definitions.

Secondary outcome measures were defined in order to evaluate the recurrence rate and the time to recurrence of diarrhea at a $25 \mathrm{mg} / \mathrm{kg}$ dosage of tylosin. The recurrence of diarrhea was defined as when the dog's feces had a consistency score of at least four on the scale for at least two consecutive defecations. Further secondary outcome measures were defined to assess the influence of the three different tylosin dosages $(5,15$ and $25 \mathrm{mg} / \mathrm{kg})$ on the time of ceasing of diarrhea. The day on which the diarrhea ceased was defined as the day of the treatment period on which the fecal score for each of the dog's feces was and remained three or less.

\section{Interventions}

When assessing the dogs' eligibility for this present dosage determination trial all dogs had diarrhea and began their tylosin treatment at a dose of $25 \mathrm{mg} / \mathrm{kg}$ once daily for seven days. The dog owners evaluated and recorded the consistency of each of their dogs' feces. On day seven of the treatment period each dog underwent a physical examination and its fecal consistency records were collected. The investigator recorded the day on which the dog's diarrhea ceased and calculated a mean fecal consistency score for each dog over the last three days of the treatment period at a tylosin dosage of $25 \mathrm{mg} / \mathrm{kg}$; the dogs defined as responders were deemed eligible to participate in the dosage determination trial. Treatment at $25 \mathrm{mg} / \mathrm{kg}$ tylosin was then discontinued for the participating dogs and a follow-up period of up to two months served to determine whether the diarrhea recurred. No additional changes were permitted in the dog's feeding management or medication. Upon meeting the criteria for the recurrence of diarrhea (fecal score $\geq 4$ for at least two consecutive defecations) each dog underwent a physical examination, during which the investigator also assessed and confirmed the fecal consistency by rectal palpation. The dogs then received tylosin (Tylosin tartrate $120 \mathrm{mg}$ and $240 \mathrm{mg}$ tablets, University Pharmacy, Helsinki, Finland; equivalent to $100 \mathrm{mg}$ and $200 \mathrm{mg}$ tylosin) once daily for seven days in doses of either $5 \mathrm{mg} / \mathrm{kg}$ or $15 \mathrm{mg} / \mathrm{kg}$.

During the seven-day treatment period, the owners continued evaluating each of their dog's stools and recorded its fecal consistency according to the guidelines. On day three, the investigator phoned the owner to assure whether the clinical condition of the dog met the criteria of the humane endpoint of the study and whether the dog had to be released from the study and treated accordingly. A humane endpoint was defined as the point at which the dog's clinical condition was poor and it suffered from excessive diarrhea. On day seven, the dog underwent a second physical examination, including rectal palpation and an evaluation of the dog's fecal consistency. The fecal consistency records were collected, and a mean fecal consistency score was calculated for each dog based on the last three days of the treatment period at the $5 \mathrm{mg} / \mathrm{kg}$ or $15 \mathrm{mg} / \mathrm{kg}$ dosages. 


\section{Allocation to treatment groups}

After the onset of diarrhea within the two-month follow-up period the dogs received an ascending study number denoting the order in which the diarrhea randomly recurred. The investigator then assigned the dogs in a 1:1 ratio to two different dosage groups - either $5 \mathrm{mg} / \mathrm{kg}$ or $15 \mathrm{mg} / \mathrm{kg}$ - receiving tylosin orally once daily for seven days. All dogs with odd study numbers were assigned to the $5 \mathrm{mg} / \mathrm{kg}$ dosage group, and those with even study numbers to the $15 \mathrm{mg} / \mathrm{kg}$ dosage group.

\section{Blinding}

The study was single-blinded and thus open to the investigator but blinded for the dog owner. The blinding procedure was carried out by labeling the $120 \mathrm{mg}$ tylosin tartrate tablet bottles as "Tylosin tart. S" and the $240 \mathrm{mg}$ tylosin tartrate tablet bottles as "Tylosin tart. V". The tablets were visually identical.

\section{Statistical methods}

Descriptive statistics were used to summarize the data. The Shapiro-Wilk test served to determine whether the data were normally distributed; non-parametric data were expressed as median and range. The Wilcoxon signed rank test was used for non-parametric data to compare the mean fecal consistency scores of the last three days of the treatment period at the $25 \mathrm{mg} / \mathrm{kg}$ tylosin dosage to the scores at the $15 \mathrm{mg} / \mathrm{kg}$ and $5 \mathrm{mg} / \mathrm{kg}$ tylosin dosages. The Mann-Whitney $U$ test was used to examine differences between the mean fecal consistency scores in the $5 \mathrm{mg} / \mathrm{kg}$ and $15 \mathrm{mg} / \mathrm{kg}$ tylosin dosage groups. To evaluate the effect of each dosage on the time until the diarrhea ceased, the Wilcoxon signed rank test and the Mann-Whitney $U$ test were used. The level of statistical significance was set at $P<0.05$; all statistical analyses were performed with a commercially available statistical software system (SPSS 18.0 for Windows).

\section{Results}

\section{Participant flow and follow-up}

Between October 2006 and May 2008, 20 client-owned dogs responding to $25 \mathrm{mg} / \mathrm{kg}$ tylosin once daily for the seven day treatment in the tylosin effectiveness trial [6] were deemed eligible to participate in the present tylosin dosage determination trial. One owner declined to participate with the dog for personal reasons, and two dogs were excluded due to other concurrent medications that violated the inclusion criteria. A total of 15 of 17 dogs relapsed and diarrhea recurred within the two-month follow up period. The results of the enrollment, allocation and response to the two different tylosin dosages appear in Figure 1. It is noteworthy that in treating their recurrent diarrhea, 14 of 15 dogs (93\%) responding to a dose of $25 \mathrm{mg} / \mathrm{kg}$ tylosin once daily also responded to the lesser dosages of $5 \mathrm{mg} / \mathrm{kg}$ or $15 \mathrm{mg} / \mathrm{kg}$ once daily for seven days. No adverse events or side effects were recorded at any of the dosages in any of the dogs. No deviations from the study protocol occurred during the trial, and no dog had to be released from the study due to excessive diarrhea or poor clinical condition.

\section{Baseline data}

The 15 dogs included in the prospective treatment trial consisted of 12 different breeds: Golden Retriever ( $\mathrm{n}=$ $3)$, German Shepherd ( $n=2)$, and one of each of the following breeds $(n=1)$ : Pumi, Rough Collie, Miniature Schnauzer, Kromfohrländer, Doberman Pinscher, Border Collie, German Shorthaired Pointer, Nova Scotia DuckTolling Retriever, Old English Sheepdog, and mixed breed. The dogs' ages at the time of enrollment ranged from seven months to four years and three months (median three years and eleven months). The weight of the dogs at the time of enrollment ranged from $10 \mathrm{~kg}$ to $37.5 \mathrm{~kg}$ (median $25.2 \mathrm{~kg}$ ). The study population included nine intact males and six females, three of which were intact.

\section{Primary outcomes}

Figure 2 shows the mean fecal consistency scores assigned during the last three days of the treatment period for each dog participating in the trial at tylosin doses of $5 \mathrm{mg} / \mathrm{kg}(\mathrm{n}=8), 15 \mathrm{mg} / \mathrm{kg}(\mathrm{n}=7)$, and $25 \mathrm{mg} /$ $\mathrm{kg}(\mathrm{n}=15)$. Figure 3 shows the minimum and maximum, the upper and lower quartiles, interquartile range and the median of the mean fecal consistency scores at all three tylosin dosages. The median of the mean fecal consistency scores from the last three days of the treatment period in the dogs receiving tylosin at the $25 \mathrm{mg} / \mathrm{kg}$ dosage was 2.5 (95\% confidence interval, 2.32; 2.65). In the dogs receiving tylosin at the $15 \mathrm{mg} / \mathrm{kg}$ dosage, the median of the mean fecal consistency scores from the last three days of the treatment period was likewise 2.5 (95\% confidence interval, $2.10 ; 3.36)$. The median of the mean fecal consistency scores from the last three days of the treatment period in the dogs receiving $5 \mathrm{mg} / \mathrm{kg}$ tylosin was 2.63 (95\% confidence interval, 2.36; 2.82). The mean fecal consistency scores for the $25 \mathrm{mg} / \mathrm{kg}$ dosage of tylosin showed no significant difference from scores for the $15 \mathrm{mg} / \mathrm{kg}$ or $5 \mathrm{mg} / \mathrm{kg}$ dosages of tylosin (Wilcoxon signed rank test, $P=0.345$, $P=0.672$ ). Nor was the difference in the mean fecal consistency scores between the dogs receiving $15 \mathrm{mg} / \mathrm{kg}$ tylosin and those receiving $5 \mathrm{mg} / \mathrm{kg}$ tylosin significant (Mann-Whitney $U$ test, asymptotic $P=0.370$ and exact, not corrected for ties $P=0.397$ ).

\section{Secondary outcomes}

At a dosage of $25 \mathrm{mg} / \mathrm{kg}$ tylosin and a treatment duration of seven days, 15 of 17 dogs relapsed, and diarrhea recurred within the two-month follow-up period on a 


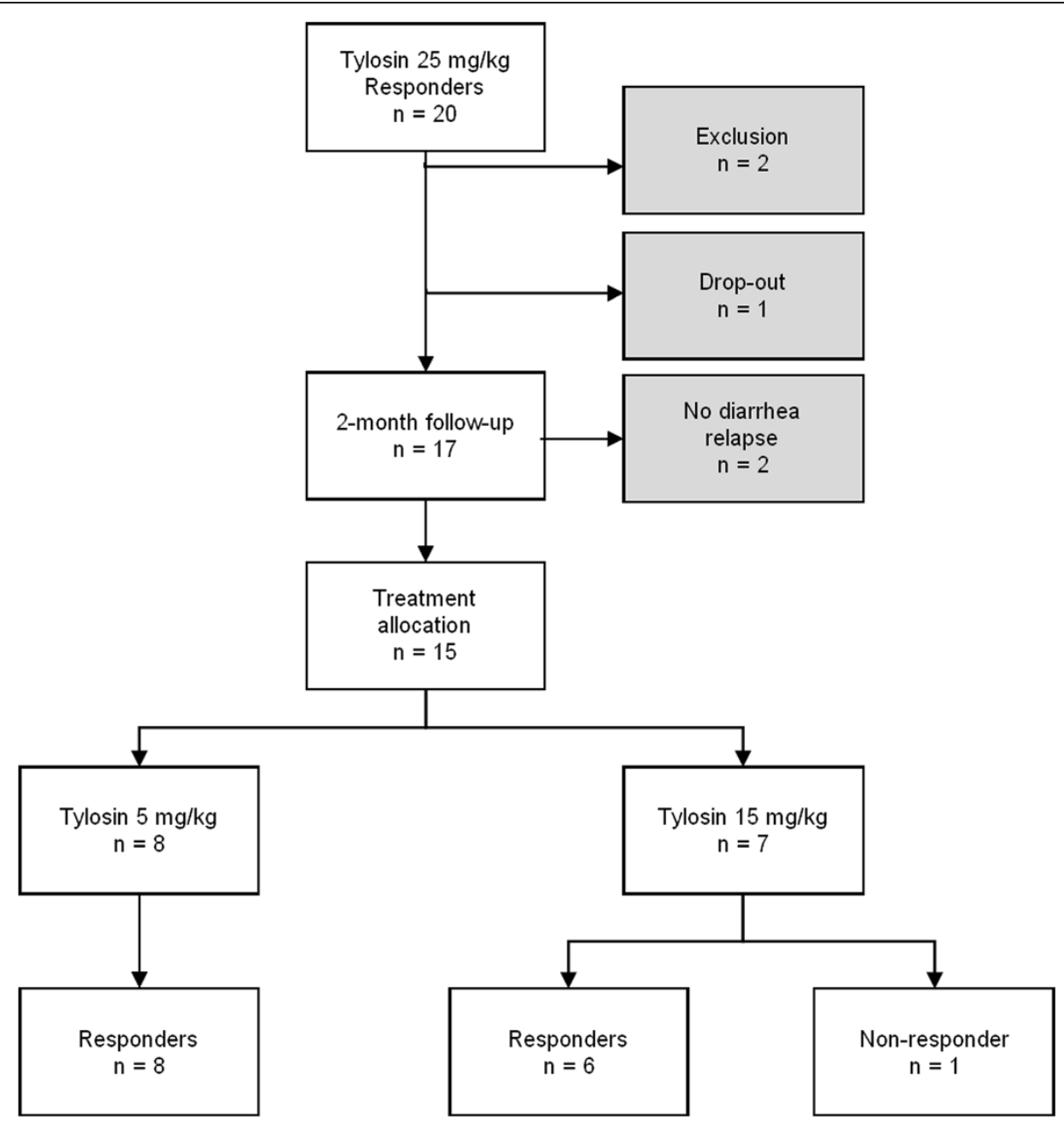

Figure 1 Flowchart of the trial. Flowchart of the results of the study enrollment, the allocation to treatment groups, and the response to the two different tylosin dosages.

median of day 9 (range $1-33$ ), resulting in a diarrhea recurrence rate of $88 \%$.

The day on which the diarrhea ceased in the dogs when receiving the $25 \mathrm{mg} / \mathrm{kg}$ dosage of tylosin $(\mathrm{n}=15)$ ranged from days 1 to 5 (median day 3; 95\% confidence interval, $2.20 ; 3.40)$. At the $15 \mathrm{mg} / \mathrm{kg}$ dosage of tylosin $(\mathrm{n}=7)$, the day on which diarrhea ceased ranged from days 2 to 5 (median day $2 ; 95 \%$ confidence interval, $1.68 ; 3.82$ ) of the treatment period, and at the $5 \mathrm{mg} / \mathrm{kg}$ dosage of tylosin $(\mathrm{n}=8)$ from days 1 to 5 (median day 2.5; 95\% confidence interval, $1.40 ; 3.94)$. The number of days until the diarrhea ceased in the dogs receiving $5 \mathrm{mg} / \mathrm{kg}$ tylosin compared to those receiving $15 \mathrm{mg} / \mathrm{kg}$ tylosin was not statistically significantly different (Mann-Whitney $U$ test, asymptotic $P=0.835$ ).

\section{Discussion}

This study was conducted to evaluate the efficacy of two low dose oral tylosin treatment regimens to control the relapse of diarrhea in dogs suffering from TRD. Our results showed that 14 of the 15 dogs (93\%) suffering from recurrent diarrhea that previously responded to oral tylosin therapy at a dose of $25 \mathrm{mg} / \mathrm{kg}$ once daily for seven days, responded to the lower doses of $5 \mathrm{mg} / \mathrm{kg}$ and $15 \mathrm{mg} / \mathrm{kg}$ once daily for seven days after diarrhea relapse. These data indicate that a suitable daily oral dose of tylosin for the treatment of diarrhea relapses in canine TRD may thus far be less than the current recommendations in textbooks, which vary from 25 to $80 \mathrm{mg} / \mathrm{kg}$ $[2,7,8]$. The limitations of this study were its rather small sample size, as well as lack of randomization and controls. Nevertheless, our results are in agreement with those of a previous TRD study in which dog owners reduced the tylosin dose for their dogs as low as possible (6-16 $\mathrm{mg} / \mathrm{kg}$ per day) while maintaining their dogs' health [5]. In both the aforementioned and the present studies, however, all dogs had previously received several oral tylosin treatments, so whether the efficacy would remain the same had the dog initially received tylosin at low dosages remains uncertain. The groups receiving $5 \mathrm{mg} / \mathrm{kg}$ and $15 \mathrm{mg} / \mathrm{kg}$ tylosin were not independent of 


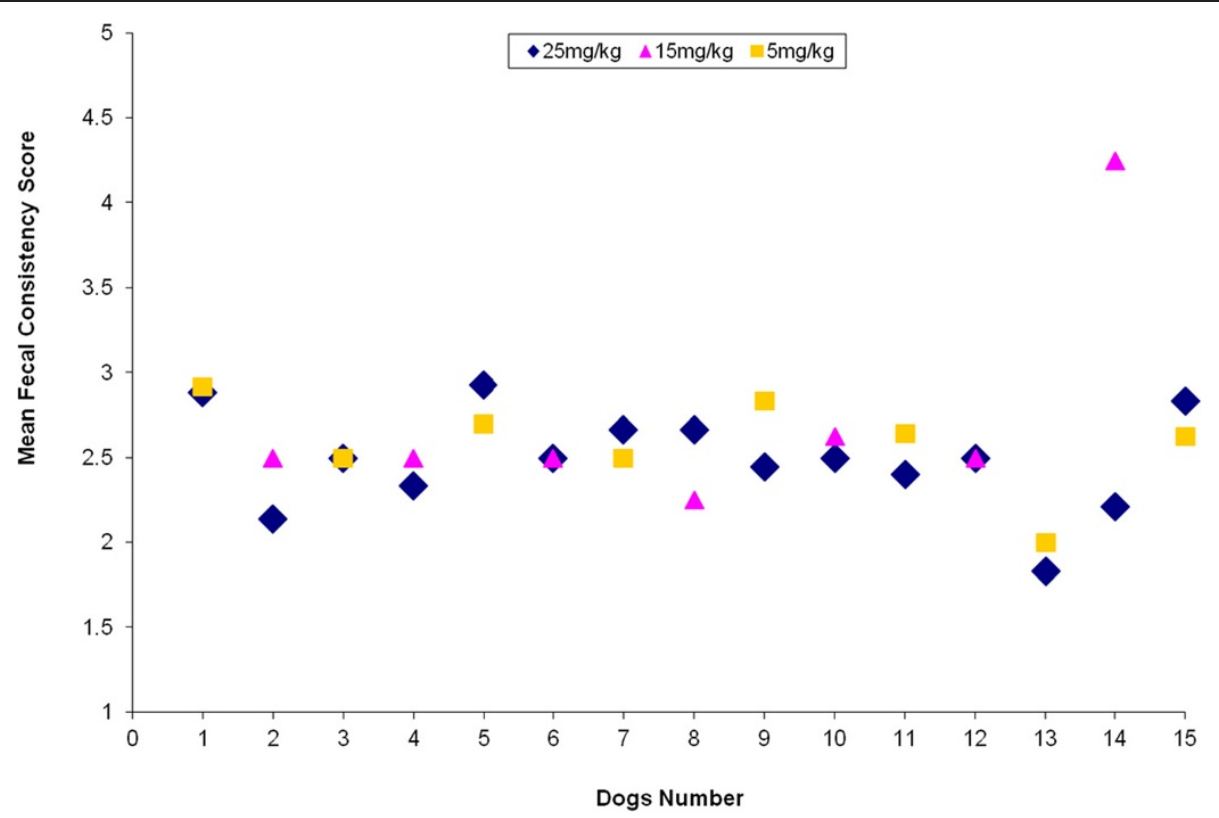

Figure 2 Mean fecal consistency scores per dog. Mean fecal consistency scores at dosages of $25 \mathrm{mg} / \mathrm{kg}(\mathrm{n}=15), 15 \mathrm{mg} / \mathrm{kg}(\mathrm{n}=7)$, and $5 \mathrm{mg} / \mathrm{kg}$ $(n=8)$ tylosin per dog assessed during the last three days of the seven-day treatment period.

the $25 \mathrm{mg} / \mathrm{kg}$ tylosin group. Still a statistical comparison was carried out to investigate whether the mean fecal consistency scores after the low dose treatments for diarrhea relapse were similar to the scores at the initial treatment and to compare only the treatment outcomes, not its efficacy.

In the present study, one dog failed to respond to the $15 \mathrm{mg} / \mathrm{kg}$ once daily dosage, but did respond the $25 \mathrm{mg} / \mathrm{kg}$ once daily dosage, possibly because this time the cause of diarrhea in this dog may have differed. Since the owners declined further diagnostic investigations in this case, the reasons for the non-response remain uncertain.
Although pharmacokinetic studies have explored the intramuscular and intravenous administration of tylosin in dogs [18], studies on per oral administration are lacking. Consequently, the dosing interval of per oral administration remains unknown. Individual authors' recommendations in textbooks for the dosing interval of tylosin differ, ranging from one to three times daily $[2,7,8]$. Our data show that a once daily dosing interval is sufficient to stop diarrhea in $14 / 15$ (93\%) of the participating dogs during the treatment period. This finding is consistent with those of earlier reports on TRD in which a once-daily dosing interval proved successful in controlling diarrhea in dogs with recurrent

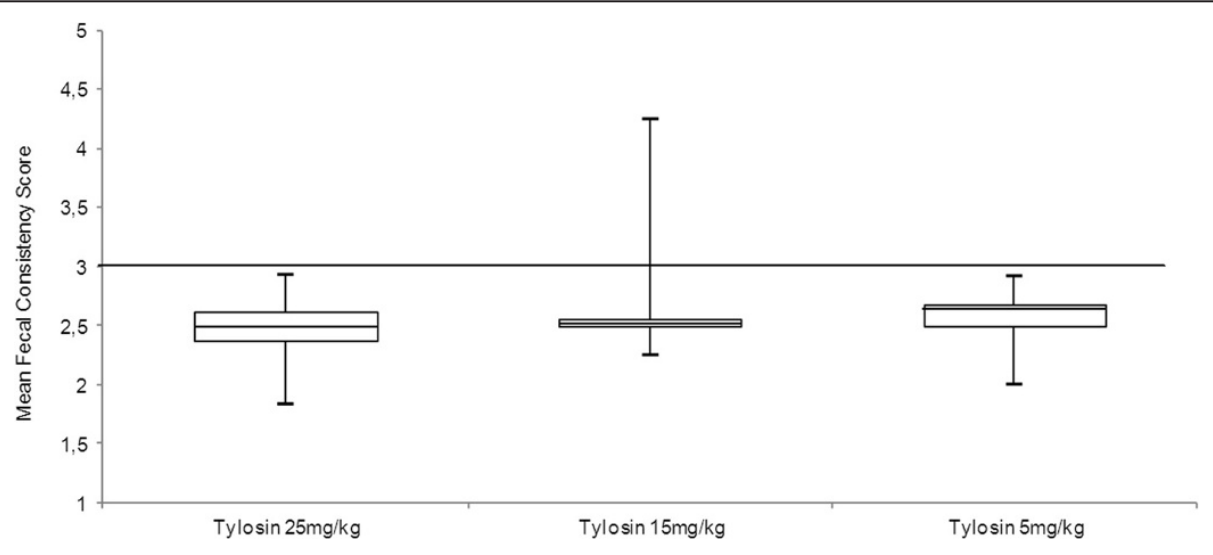

Figure 3 Box and whisker plots of the mean fecal consistency scores. Box and whisker plots of the mean fecal consistency scores assessed during the last three days of the seven-day treatment period at tylosin dosages of $25 \mathrm{mg} / \mathrm{kg}(\mathrm{n}=15), 15 \mathrm{mg} / \mathrm{kg}(\mathrm{n}=7)$ and $5 \mathrm{mg} / \mathrm{kg}(\mathrm{n}=8)$. The limits of the box represent the $25^{\text {th }}$ and $75^{\text {th }}$ percentile values, the line within the box represents the median values and the whiskers represent the range. The black line indicates the cut-off value for a responder, set at a score of 3 on the mean fecal consistency score scale. 
diarrhea $[5,6,15]$, and shows that a more frequent dosing interval is unnecessary in the treatment of diarrhea relapses in TRD dogs. A once daily dosing makes the treatment cost effective and more convenient for both the dog and the owner.

Controversy also surrounds recommendations regarding the duration of tylosin therapy in chronic enteropathies in dogs. So far, recommendations for the duration of antibiotic treatment in antibiotic-responsive diarrhea can range up to six weeks, because premature cessation of treatment could lead to relapse [1]. However, to the best of our knowledge, no studies have yet investigated whether the treatment duration affects the recurrence rate. All of the dogs in this present trial received tylosin for seven days at a dosage of $25 \mathrm{mg} / \mathrm{kg}$ before it was terminated. During the two month follow-up $88 \%$ of the dogs relapsed. This is a higher recurrence rate than the one in our previous study, where diarrhea recurred in only $43.3 \%$ of the dogs investigated [6]. In the aforementioned study, the duration of tylosin treatment varied between the dogs. However, the high prevalence of recurrence is in accordance with that of an earlier report on TRD in which the low dosage tylosin treatment lasted for at least six weeks. When the treatment was terminated, the diarrhea recurred within 30 days in $85.7 \%$ of the dogs [5]. Since an at least six-week tylosin course in TRD dogs has not lead to smaller relapse rates than a seven-day course, we conclude that a seven-day treatment period may prove sufficient in the treatment of TRD in dogs. One limitation of this study was that we were unable to follow the dogs beyond the end of this trial to determine whether, after receiving the low dosages, they showed signs of diarrhea relapse and whether the time interval between the cessation of treatment and the relapse of clinical signs depended on the dose or duration of the treatment. Additional studies are warranted to further assess the effect of treatment duration on diarrhea relapse.

At a dosage of $25 \mathrm{mg} / \mathrm{kg}$ tylosin and treatment duration of seven days diarrhea recurred in the dogs of this trial on a median of day 9, which is in accordance with a former study on TRD where at tylosin dosages varying individually from 6 to $16 \mathrm{mg} / \mathrm{kg}$, the diarrhea reappeared on a median day of 7 [5].

Until now, some have suggested that in dogs with antibiotic-responsive diarrhea who have shown a suboptimal response, the antibiotic should be changed after the first two weeks [1]. In the present study the diarrhea ceased in all dogs by day five of the tylosin treatment. The diarrhea ceased in the dogs receiving 5,15 and $25 \mathrm{mg} / \mathrm{kg}$ tylosin on a median day of 2.5, 2 and 3, respectively. This result falls within the same time frame as those published in earlier studies on TRD $[5,15]$ and indicates that in the absence of a response after a few days of tylosin treatment, the treatment should be terminated and further diagnostics introduced as soon as possible. In small animal practice the uncritical use of antibiotics, including tylosin, contributes to the development of resistant intestinal bacterial flora which also poses a risk to public health. Thus, until alternative treatment options are found, one should aim to restrict the usage of tylosin in chronic enteropathies in dogs when systemic disorders have been ruled out and, despite thorough investigations, no other underlying primary intestinal disorder is evident.

\section{Conclusions}

In conclusion, our results indicate that a suitable oral dosage of tylosin in the treatment of diarrhea relapses in canine tylosin-responsive diarrhea could be as low as $5 \mathrm{mg} / \mathrm{kg}$ once daily for seven days.

\section{Abbreviation}

TRD: Tylosin-responsive diarrhea.

\section{Competing interests}

The authors declare that they have no competing interests.

\section{Authors' contributions}

SK made the practical arrangements, carried out the clinical examinations and data collection and drafted the manuscript. TS made a substantial contribution to the design of the study and helped draft the manuscript. EW made a substantial contribution in the design of the study and assisted significantly in drafting the manuscript. All authors read and approved the final manuscript.

\section{Acknowledgements}

We acknowledge Dr. Katja Mustonen and Dr. Nina Menna of Vetcare for their cooperation during the study as well as Dr. Merja Rantala for improving the manuscript with her constructive comments.

The study was funded by Vetcare Ltd., Mäntsälä, Finland and supported by both the Finnish Veterinary Foundation and the Finnish Foundation of Veterinary Research.

Part of the work was presented as an abstract at the 2010 ACVIM Forum, Comparative Gastroenterology Society Session, Anaheim, California, USA.

\section{Received: 22 October 2013 Accepted: 23 June 2014}

Published: 6 August 2014

\section{References}

1. Hall EJ: Antibiotic-responsive diarrhea in small animals. Vet Clin North Am Small Anim Pract 2011, 41:273-286.

2. Hall EJ, German AJ: Diseases of the Small Intestine. In Textbook of Veterinary Internal Medicine. 7th edition. Edited by Ettinger SJ, Feldman EC. St Louis, MO: Elsevier Saunders; 2010:1526-1572.

3. Van Kruiningen $\mathrm{HJ}$ : Clinical efficacy of tylosin in canine inflammatory bowel disease. J Am Anim Hosp Assoc 1976, 12:498-501.

4. Westermarck E, Myllys V, Aho M: Effect of treatment on the jejunal and colonic bacterial flora of dogs with exocrine pancreatic insufficiency. Pancreas 1993, 8:559-562.

5. Westermarck E, Skrzypczak T, Harmoinen J, Steiner JM, Ruaux CG, Williams DA, Eerola E, Sundbäck P, Rinkinen M: Tylosin-responsive chronic diarrhea in dogs. J Vet Intern Med 2005, 19:177-186.

6. Kilpinen S, Spillmann T, Syrjä P, Skrzypczak T, Louhelainen M, Westermarck E: Effect of tylosin on dogs with suspected tylosin-responsive diarrhea: $A$ placebo-controlled, randomized, double-blinded, prospective clinical trial. Acta Vet Scand 2011, 52:26.

7. Westermarck E: Tylosin Responsive Diarrhea. In Kirk's Current Veterinary Therapy. 14th edition. Edited by Bonagura JD, Twedt DC. St Louis, MO: Elsevier Saunders; 2009:506-509.

8. Sherding RG, Johnson SE: Diseases of the Intestines. In Saunders Manual of Small Animal Practice. 3rd edition. Edited by Birchard SJ, Sherding RG. St. Louis, Mo: Saunders; 2006:702-738. 
9. Cockcroft P, Holmes M: Handbook of Evidence-based Veterinary Medicine. Oxford, UK: Blackwell Publishing Ltd; 2003.

10. Begg C, Cho M, Eastwood S, Horton R, Moher D, Olkin I, Pitkin R, Rennie D, Schulz KF, Simel D, Stroup DF: Improving the quality of reporting of randomized controlled trials: The CONSORT statement. J Am Med Assoc 1996, 276:637-639

11. Moher D, Schulz KF, Altman DG, Lepage L: The CONSORT statement: revised recommendations for improving the quality of reports of parallel-group randomised trials. Lancet 2001, 357:1191-1194.

12. Sargeant JM, Thompson A, Valcour J, Elgie R, Saint-Onge J, Marcynuk P, Snedeker K: Quality of reporting of clinical trials of dogs and cats and associations with treatment effects. J Vet Intern Med 2010, 24:44-50.

13. Schulz KF, Altman DG, Moher D, CONSORT G: CONSORT 2010 statement: updated guidelines for reporting parallel group randomised trials. BMJ 2010, 340:332

14. Moxham G: The WALTHAM faeces scoring system - a tool for veterinarians and pet owners: how does your pet rate? WALTHAM Focus 2001, 11:24-25.

15. Westermarck E, Frias R, Skrzypczak T: Effect of diet and tylosin on chronic diarrhea in beagles. J Vet Intern Med 2005, 19:822-827.

16. Blackwood RS, Tarara RP, Christe KL, Spinner A, Lerche NW: Effects of the macrolide drug tylosin on chronic diarrhea in rhesus macaques (Macaca mulatta). Comp Med 2008, 58:81-87.

17. Bybee SN, Scorza AV, Lappin MR: Effect of the probiotic Enterococcus faecium SF68 on presence of diarrhea in cats and dogs housed in an animal shelter. J Vet Intern Med 2011, 25:856-860.

18. Weisel MK, Powers JD, Powers TE, Baggot JD: A pharmacokinetic analysis of tylosin in the normal dog. Am J Vet Res 1977, 38:273-275.

doi:10.1186/s13028-014-0043-5

Cite this article as: Kilpinen et al:: Efficacy of two low-dose oral tylosin regimens in controlling the relapse of diarrhea in dogs with tylosin-responsive diarrhea: a prospective, single-blinded, two-arm parallel, clinical field trial. Acta Veterinaria Scandinavica 2014 56:43.

\section{Submit your next manuscript to BioMed Central and take full advantage of:}

- Convenient online submission

- Thorough peer review

- No space constraints or color figure charges

- Immediate publication on acceptance

- Inclusion in PubMed, CAS, Scopus and Google Scholar

- Research which is freely available for redistribution 